\title{
Hydrodynamic models of type I X-ray bursts: metallicity effects
}

\author{
Jordi José ${ }^{1}$ \\ Dept. Física i Enginyeria Nuclear, Universitat Politècnica de Catalunya (UPC), and Institut d'Estudis \\ Espacials de Catalunya (IEEC), C. Urgell 187, EUETIB, E-08036 Barcelona, Spain \\ E-mail: jordi.jose@upc.edu
}

\section{Fermín Moreno}

Dept. Física i Enginyeria Nuclear, Universitat Politècnica de Catalunya (UPC)

C. Urgell 187, EUETIB, E-08036 Barcelona, Spain

E-mail: morenodieec.fcr.es

\section{Anuj Parikh}

Dept. Física i Enginyeria Nuclear, Universitat Politècnica de Catalunya (UPC)

C. Urgell 187, EUETIB, E-08036 Barcelona, Spain

E-mail: xrayburstegmail.com

\section{Christian Iliadis}

Dept. of Physics and Astronomy, University of North Carolina, Chapel Hill, NC, and Triangle

Universities Nuclear Laboratory, Durham, NC, 174 Phillips Hall, Chapel Hill, NC 27599-3255 ,USA

E-mail: iliadis@unc.edu

Type I X-ray bursts are thermonuclear stellar explosions driven by charged-particle reactions. Detailed analysis of the relevant reactions along the main nuclear path has only been scarcely addressed, mainly in the context of parameterized one-zone models. Here, we present a detailed study of the nucleosynthesis and nuclear processes powering type I X-ray bursts. The reported bursts have been computed by means of a spherically symmetric (1D), Lagrangian, hydrodynamic code, linked to a nuclear reaction network that contains 325 isotopes (from ${ }^{1} \mathrm{H}$ to

${ }^{107} \mathrm{Te}$ ), and 1392 nuclear processes. These evolutionary sequences, followed from the onset of accretion up to the explosion and expansion stages, have been performed for 2 different metallicities to explore the dependence between the extension of the main nuclear flow and the initial metal content. We carefully analyze the physical parameters that determine the light curve (including recurrence times, ratios between persistent and burst luminosities, or the extent of the envelope expansion). Results are in qualitative agreement with the observed properties of some well-studied bursting sources.

11th Symposium on Nuclei in the Cosmos - NIC XI

Heidelberg, Germany

July 19-232010

\footnotetext{
${ }^{1}$ Speaker
} 


\section{Introduction}

Type I X-Ray Bursts (XRBs) are thermonuclear stellar explosions driven by chargedparticle reactions. In the regime for combined H/He-ignition, the main nuclear flow is dominated by the $\mathrm{r} p$-process (rapid proton-captures and $\beta^{+}$-decays), the $3 \alpha$-reaction, and the $\alpha p$ process (a suite of $(\alpha, p)$ and $(p, \gamma)$ reactions). The main flow is expected to proceed away from the valley of stability, eventually reaching the proton drip line beyond $A=38$.

Detailed nucleosynthetic calculations, with extended networks, have been performed under different approaches: one-zone models (Schatz et al. 1998, 2001 -up to Xe-, Koike et al. 2004 up to Bi-), and 1-D hydrodynamic models (Woosley et al. 2004; Fisker et al. 2008; José et al. 2010 -up to the SnSbTe mass region-). Here, we will report on recent, 1-D hydrodynamic models, emphasizing the role played by the metallicity of the accreted material on the burst properties.

\begin{tabular}{|lcccccc|}
\hline \multicolumn{7}{|c|}{ Model 1 } \\
\hline \hline Burst & $T_{\text {peak }}(\mathrm{K})$ & $t\left(T_{\text {peak }}\right)(\mathrm{s})$ & $\tau_{\text {rec }}(\mathrm{hr})$ & $L_{\text {peak }}\left(L_{\odot}\right)$ & $\tau_{0.01}(\mathrm{~s})$ & $\alpha$ \\
\hline 1 & $1.06 \times 10^{9}$ & 21192 & 5.9 & $9.7 \times 10^{4}$ & 75.8 & 60 \\
2 & $1.15 \times 10^{9}$ & 44342 & 6.4 & $1.7 \times 10^{5}$ & 62.3 & 40 \\
3 & $1.26 \times 10^{9}$ & 62137 & 4.9 & $2.1 \times 10^{5}$ & 55.4 & 34 \\
4 & $1.12 \times 10^{9}$ & 80568 & 5.1 & $1.2 \times 10^{5}$ & 75.7 & 36 \\
\hline & & & Model 3 & & & \\
\hline \hline Burst & $T_{\text {peak }}(\mathrm{K})$ & $t\left(T_{\text {peak }}\right)(\mathrm{s})$ & $\tau_{\text {rec }}(\mathrm{hr})$ & $L_{\text {peak }}\left(L_{\odot}\right)$ & $\tau_{0.01}(\mathrm{~s})$ & $\alpha$ \\
\hline 1 & $1.40 \times 10^{9}$ & 65110 & 18.1 & $1.0 \times 10^{5}$ & 423 & 34 \\
2 & $1.39 \times 10^{9}$ & 98879 & 9.4 & $1.1 \times 10^{5}$ & 296 & 24 \\
3 & $1.32 \times 10^{9}$ & 130816 & 8.9 & $9.8 \times 10^{4}$ & 281 & 24 \\
4 & $1.30 \times 10^{9}$ & 162777 & 8.9 & $1.0 \times 10^{5}$ & 252 & 27 \\
5 & $1.26 \times 10^{9}$ & 194266 & 8.8 & $1.0 \times 10^{5}$ & 250 & 30 \\
\hline
\end{tabular}

Table 1. Summary of bursts properties for model 1 (a $1.4 \mathrm{M}_{\odot}$ neutron star accreting solar-like matter $[\mathrm{Z}=0.02]$ at a rate $\mathrm{M}_{\mathrm{acc}}=1.75 \times 10^{-9} \mathrm{M}_{\odot} \mathrm{yr}^{-1}$ ), and model 3 (same as model 1 , with $\mathrm{Z}=10^{-3}$ ).

\section{Nucleosynthesis in type I X-ray bursts}

In this work, we report a study of the nucleosynthesis and nuclear processes powering type I XRBs. The successive bursting episodes (see Table 1) have been computed by means of the spherically symmetric (1-D), Lagrangian, hydrodynamic code SHIVA, linked to a nuclear reaction network that contains 325 isotopes (from ${ }^{1} \mathrm{H}$ to ${ }^{107} \mathrm{Te}$ ), and 1392 nuclear processes. The evolutionary sequences, followed from the onset of accretion up to the explosion and expansion stages, have been computed for two different metallicities to explore the dependence between the extension of the main nuclear flow and the initial metal content. Results reveal a dependence 
of burst properties on the metallicity of the accreted material: the smaller the metal content, the larger the recurrence time, $\tau_{\text {rec }}$ (and the smaller the ratio between persistent and burst luminosities, $\alpha$ ). In turn, explosions in metal-deficient envelopes (i.e., model 3) are characterized by lower peak luminosities and longer decline times, in agreement with the pattern described in Woosley et al. (2004) and Heger et al. (2007). We found a much larger effect played by the metallicity of the accreted material as compared with Woosley et al. (2004), who explained the moderate effect as due to compositional inertia washing out the influence of the initial metallicity. Another difference concerns the extremely large differences in the gross physical characteristics - nucleosynthesis, energies, or recurrence times - between the first and subsequent bursts, as reported by Woosley et al. (2004). Indeed, the mean, mass-averaged composition of the envelope at the end of the $4^{\text {th }}$ burst computed for model 1 is dominated by ${ }^{60} \mathrm{Ni}[31 \%],{ }^{64} \mathrm{Zn}[13 \%]$, unburnt ${ }^{4} \mathrm{He}[10 \%],{ }^{32} \mathrm{~S}[9 \%],{ }^{56} \mathrm{Ni}[6 \%],{ }^{68} \mathrm{Ge}[5 \%]$, unburnt ${ }^{1} \mathrm{H} \mathrm{[4 \% ]}$, ${ }^{12} \mathrm{C},{ }^{34} \mathrm{~S},{ }^{72} \mathrm{Se}[2 \%],{ }^{28} \mathrm{Si},{ }^{35} \mathrm{Cl},{ }^{36} \mathrm{Ar},{ }^{39} \mathrm{~K}$, and ${ }^{52} \mathrm{Fe}[1 \%]$; in contrast, the ashes of the $4{ }^{\text {th }}$ burst computed for model 3, are dominated by the presence of ${ }^{105} \mathrm{Ag}[14 \%],{ }^{64} \mathrm{Zn},{ }^{104} \mathrm{Pd}[7 \%]$, unburnt ${ }^{1} \mathrm{H},{ }^{68} \mathrm{Ge}[6 \%],{ }^{60} \mathrm{Ni},{ }^{94} \mathrm{Tc}[5 \%], 4 \mathrm{He}[4 \%],{ }^{72} \mathrm{Se},{ }^{103} \mathrm{Ag}[3 \%],{ }^{76} \mathrm{Kr},{ }^{86} \mathrm{Zr},{ }^{89} \mathrm{Nb},{ }^{99} \mathrm{Rh},{ }^{101,102} \mathrm{Pd}$ [2\%], ${ }^{65} \mathrm{Zn},{ }^{69} \mathrm{Ge},{ }^{80,82,83} \mathrm{Sr},{ }^{91} \mathrm{Nb}$, and ${ }^{95,97,98} \mathrm{Ru}[1 \%]$ (with ${ }^{12} \mathrm{C} \sim 0.1 \%$ ). It is worth noting that in both models not enough ${ }^{12} \mathrm{C}$ is left to power superbursts.

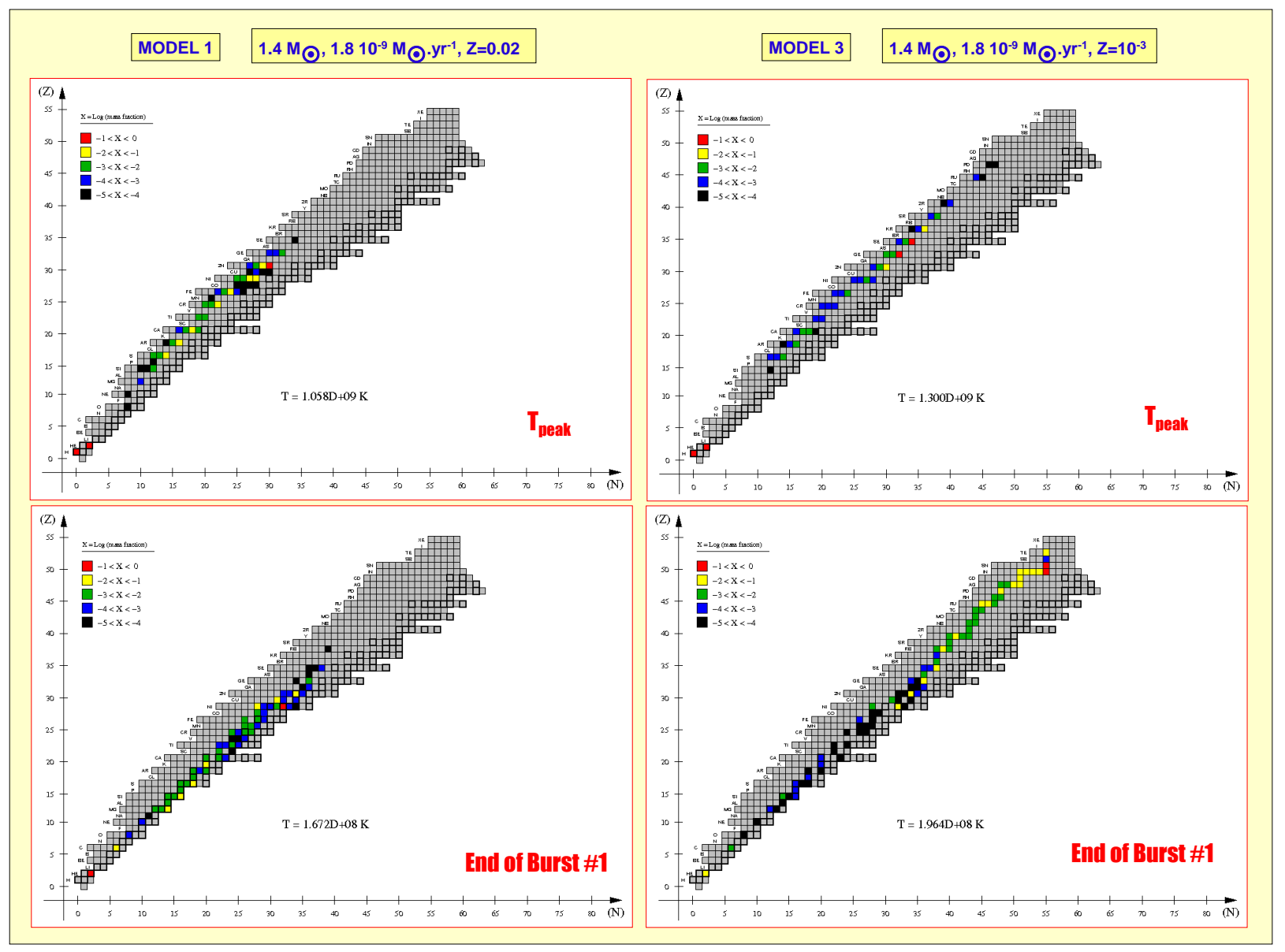

Fig. 1. The main nuclear activity, shown in terms of most abundant species, for models 1 and 3 , at $\mathrm{T}_{\text {peak }}$ (upper panels) and at the end of the first burst (lower panels). 
The burst properties summarized in Table 1 qualitatively agree with those inferred from the XRB sources GS 1826-24 [ $\left.\tau_{\text {rec }}=5.74 \pm 0.13 \mathrm{hr}, \alpha=41.7 \pm 1.6\right], 4 \mathrm{U} 1323-62\left[\tau_{\mathrm{rec}}=5.3 \mathrm{hr}, \alpha=38 \pm 4\right]$, or $4 \mathrm{U} 1608-52\left[\tau_{\mathrm{rec}}=4.14-7.5 \mathrm{hr}, \alpha=41-54\right]$, for model 1 , and $1 \mathrm{~A} 1905+00\left[\tau_{\mathrm{rec}}=8.9 \mathrm{hr}\right], 4 \mathrm{U}$ $1254-69\left[\tau_{\text {rec }}=9.2 \mathrm{hr}\right]$, or XTE J1710-281 $\left[\tau_{\text {rec }}=8.9 \mathrm{hr}, \alpha=22-100\right]$, for model 3 (see Galloway et al. 2008).

\begin{tabular}{|c|c|}
\hline Reaction & Models Affected \\
\hline${ }^{12} \mathrm{C}(\alpha, \gamma){ }^{16} \mathrm{O}^{\mathrm{a}} \ldots \ldots \ldots$ & F08, K04-B2, K04-B4, K04-B5 \\
\hline${ }^{18} \mathrm{Ne}(\alpha, p)^{21} \mathrm{Na}^{\mathrm{a}} \ldots \ldots \ldots \ldots \ldots \ldots \ldots \ldots \ldots \ldots \ldots \ldots \ldots$ & $\mathrm{K} 04-\mathrm{B} 1^{\mathrm{b}}$ \\
\hline 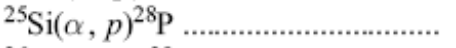 & K04-B5 \\
\hline${ }^{26 g} \mathrm{Al}(\alpha, p){ }^{29} \mathrm{Si}$ & F08 \\
\hline$\left.{ }^{29} \mathrm{~S}(\alpha, p)\right)^{32} \mathrm{Cl} \ldots \ldots \ldots \ldots \ldots \ldots \ldots \ldots$ & K04-B5 \\
\hline 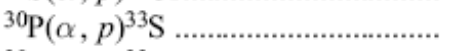 & K04-B4 \\
\hline 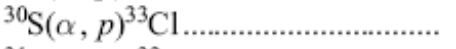 & $\mathrm{K} 04-\mathrm{B} 4,^{\mathrm{b}} \mathrm{K} 04-\mathrm{B} 5^{\mathrm{b}}$ \\
\hline${ }^{31} \mathrm{Cl}(p, \gamma){ }^{32} \mathrm{Ar} \ldots \ldots \ldots \ldots \ldots \ldots \ldots \ldots \ldots \ldots \ldots \ldots \ldots$ & K04-B1 \\
\hline 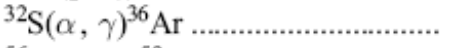 & K04-B2 \\
\hline 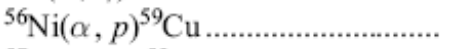 & S01, ${ }^{\mathrm{b}} \mathrm{K} 04-\mathrm{B} 5$ \\
\hline 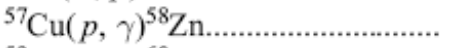 & F08 \\
\hline${ }^{59} \mathrm{Cu}(p, \gamma){ }^{60} \mathrm{Zn}$ & S01, ${ }^{\mathrm{b}} \mathrm{K} 04-\mathrm{B} 5$ \\
\hline 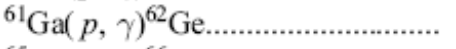 & F08, K04-B1, K04-B2, K04-B5, K04-B6 \\
\hline 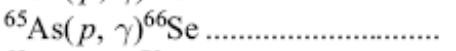 & $\mathrm{K} 04,^{\mathrm{b}} \mathrm{K} 04-\mathrm{B} 1, \mathrm{~K} 04-\mathrm{B} 2,{ }^{\mathrm{b}} \mathrm{K} 04-\mathrm{B} 3{ }^{\mathrm{b}}{ }^{\mathrm{b}} \mathrm{K} 04-\mathrm{B} 4, \mathrm{~K} 04-\mathrm{B} 5, \mathrm{~K} 04-\mathrm{B} 6$ \\
\hline${ }^{69} \mathrm{Br}(p, \gamma)^{70} \mathrm{Kr}$ & K04-B7 \\
\hline 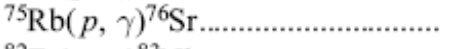 & K04-B2 \\
\hline${ }^{82} \operatorname{Zr}(p, \gamma){ }^{83} \mathrm{Nb} \ldots \ldots \ldots \ldots \ldots \ldots \ldots \ldots \ldots \ldots$ & K04-B6 \\
\hline${ }^{84} \mathrm{Zr}(p, \gamma)^{85} \mathrm{Nb}$ & K04-B2 \\
\hline${ }^{84} \mathrm{Nb}(p, \gamma){ }^{85} \mathrm{Mo}$ & K04-B6 \\
\hline${ }^{85} \mathrm{Mo}(p, \gamma)^{86} \mathrm{Tc}$ & F08 \\
\hline${ }^{86} \mathrm{Mo}(p, \gamma)^{87} \mathrm{Tc}$ & F08, K04-B6 \\
\hline${ }^{87} \mathrm{Mo}(p, \gamma)^{88} \mathrm{Tc}$ & K04-B6 \\
\hline${ }^{92} \mathrm{Ru}(p, \gamma){ }^{93} \mathrm{Rh}$ & K04-B2, K04-B6 \\
\hline${ }^{93} \operatorname{Rh}(p, \gamma){ }^{94} \mathrm{Pd}$ & K04-B2 \\
\hline${ }^{96} \mathrm{Ag}(p, \gamma){ }^{97} \mathrm{Cd}$ & K04, K04-B2, K04-B3, K04-B7 \\
\hline${ }^{102} \operatorname{In}(p, \gamma)^{103} \mathrm{Sn}$ & K04, K04-B3 \\
\hline${ }^{103} \operatorname{In}(p, \gamma)^{104} \mathrm{Sn}$ & K04-B3, K04-B7 \\
\hline${ }^{103} \mathrm{Sn}(\alpha, p){ }^{106} \mathrm{Sb}$ & $\mathrm{S} 01^{\mathrm{b}}$ \\
\hline
\end{tabular}

Table 2. Summary of the most influential nuclear processes for XRB nucleosynthesis (see Parikh et al. 2008, for details).

\section{Nuclear uncertainties}

Available reaction rates for XRB nucleosynthesis calculations often rely on theoretical Hauser-Feschbach estimates, and hence are affected by some uncertainty. Recent studies (Parikh et al. 2008, 2009) have identified the main sources of uncertainty from the several thousand nuclear processes that can be considered in XRB nucleosynthesis calculations: the number of key reactions is very limited ( 30 reactions; Table 2$)$, with the most influential ones for XRB conditions being ${ }^{65} \mathrm{As}(\mathrm{p}, \gamma)$ and ${ }^{61} \mathrm{Ga}(\mathrm{p}, \gamma)$. Mass measurements around the waiting point nuclei are also requested to improve the corresponding nucleosynthetic predictions. Indeed, experimental determinations of the masses of ${ }^{62} \mathrm{Ge},{ }^{65} \mathrm{As},{ }^{66} \mathrm{Se},{ }^{69} \mathrm{Br},{ }^{70} \mathrm{Kr},{ }^{84} \mathrm{Nb},{ }^{85} \mathrm{Mo},{ }^{86,87} \mathrm{Tc}$, 
${ }^{96} \mathrm{Ag},{ }^{97} \mathrm{Cd},{ }^{103} \mathrm{Sn}$, and ${ }^{106} \mathrm{Sb}$ are lacking. Better precision for the experimentally-known masses of ${ }^{71} \mathrm{Br},{ }^{83} \mathrm{Nb}$, and ${ }^{86} \mathrm{Mo}$ may be required as these are known to only $\pm 568,315$, and $438 \mathrm{keV}$,

\begin{tabular}{|c|c|}
\hline Reaction & Models Affected \\
\hline${ }^{15} \mathrm{O}(\alpha, \gamma){ }^{19} \mathrm{Ne}^{\mathrm{a}} \ldots$ & K04, K04-B1, K04-B \\
\hline${ }^{18} \mathrm{Ne}(\alpha, p)^{21} \mathrm{Na}^{\mathrm{a}} \ldots \ldots \ldots \ldots \ldots$ & K04-B1, K04-B6 \\
\hline${ }^{22} \mathrm{Mg}(\alpha, p)^{25} \mathrm{Al} \ldots \ldots \ldots \ldots \ldots$ & F08 \\
\hline${ }^{23} \mathrm{Al}(p, \gamma)^{24} \mathrm{Si} \ldots \ldots \ldots \ldots \ldots$ & K04-B1 \\
\hline${ }^{24} \mathrm{Mg}(\alpha, p)^{27} \mathrm{Al}^{\mathrm{a}} \ldots \ldots \ldots \ldots \ldots$ & K04-B2 \\
\hline${ }^{269} \mathrm{Al}(p, \gamma)^{27} \mathrm{Si}^{\mathrm{a}} \ldots \ldots \ldots \ldots \ldots$ & F08 \\
\hline${ }^{28} \mathrm{Si}(\alpha, p)^{31} \mathrm{P}^{\mathrm{a}} \ldots \ldots \ldots \ldots \ldots \ldots$ & K04-B4 \\
\hline${ }^{30} \mathrm{~S}(\alpha, p)^{33} \mathrm{Cl} \ldots \ldots \ldots \ldots \ldots \ldots$ & K04-B4, K04-B5 \\
\hline${ }^{31} \mathrm{Cl}(p, \gamma)^{32} \mathrm{Ar} \ldots \ldots \ldots \ldots \ldots \ldots$ & K04-B3 \\
\hline${ }^{32} \mathrm{~S}(\alpha, p)^{35} \mathrm{Cl} \ldots \ldots \ldots \ldots \ldots \ldots$ & K04-B2 \\
\hline${ }^{35} \mathrm{Cl}(p, \gamma)^{36} \mathrm{Ar}^{\mathrm{a}} \ldots \ldots \ldots \ldots \ldots$ & K04-B2 \\
\hline${ }^{56} \mathrm{Ni}(\alpha, p)^{59} \mathrm{Cu} \ldots \ldots \ldots \ldots \ldots$ & S01 \\
\hline${ }^{59} \mathrm{Cu}(p, \gamma)^{60} \mathrm{Zn} \ldots \ldots \ldots \ldots \ldots$ & S01 \\
\hline 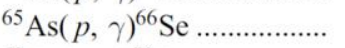 & K04, K04-B2, K04-B3 \\
\hline${ }^{69} \operatorname{Br}(p, \gamma)^{70} \mathrm{Kr} \ldots \ldots \ldots \ldots$ & S01 \\
\hline${ }^{71} \mathrm{Br}(p, \gamma)^{72} \mathrm{Kr} \ldots \ldots \ldots \ldots \ldots$ & K04-B7 \\
\hline${ }^{103} \mathrm{Sn}(\alpha, p)^{106} \mathrm{Sb} \ldots \ldots \ldots \ldots$ & S01 \\
\hline
\end{tabular}
respectively. The mass of ${ }^{65} \mathrm{As}$ is particularly critical in this context, since variation of the ${ }^{65} \mathrm{As}(\mathrm{p}, \gamma){ }^{66} \mathrm{Se}$ rate led to significant effects in most of the XRB models considered. Measurements of the mass of ${ }^{66} \mathrm{Se}\left(t_{1 / 2}=33 \mathrm{~ms}\right)$ as well as spectroscopy of ${ }^{66} \mathrm{Se}$ are also needed to improve our knowledge of the important ${ }^{65} \mathrm{As}(\mathrm{p}, \gamma)$ reaction rate in XRBs (Parikh et al. 2009). Other nuclear uncertainties affect nuclear process whose variation has an imprint in the overall energy output: this includes ${ }^{31} \mathrm{~S}(\mathrm{p}, \gamma),{ }^{35} \operatorname{Ar}(\mathrm{p}, \gamma)$, ${ }^{27} \mathrm{Si}(\mathrm{p}, \gamma),{ }^{14} \mathrm{O}(\alpha, \gamma)$, and the list of reactions compiled in Table 3 .

Table 3. Summary of the most influential nuclear processes affecting the energy output in XRB nucleosynthesis studies (see Parikh et al. 2008, for details).

Another study on the impact of nuclear uncertainties on XRB nucleosynthesis calculations (Cyburt et al. 2010) was published after completion of the work reported in this paper.

\section{References}

[1] R. H. Cyburt, et al., The JINA REACLIB Database: Its Recent Updates and Impact on Type-I X-ray Bursts, ApJS 189 (2010), 240.

[2] J.L. Fisker, H. Schatz, \& F.-K. Thielemann, Explosive hydrogen burning during type I X-ray bursts, ApJS 174 (2008), 261.

[3] D.K. Galloway, M.P. Muno, J.M. Hartman, D. Psaltis, \& D. Chakrabarty, Thermonuclear (Type I) X-Ray Bursts Observed by the Rossi X-Ray Timing Explorer, ApJS 179 (2008), 360.

[4] A. Heger, A. Cumming, D.K. Galloway, \& S.E. Woosley, Models of type I X-ray bursts from GS 1826-24: a probe of rp-process hydrogen burning, ApJ 671 (2007), L41.

[5] J. José, F. Moreno, A. Parikh, \& C. Iliadis, Hydrodynamic models of type I X-ray bursts: metallicity effects, ApJS 189 (2010), 204.

[6] O. Koike, et al., Final products of the rp-process on accreting neutron stars, ApJ 603 (2004), 242.

[7] A. Parikh, J. José, M. Moreno, \& C. Iliadis, The effects of variations in nuclear processes on type I $X$-ray burst nucleosynthesis, ApJS 178 (2008), 110.

[8] A. Parikh, J. José, M. Moreno, C. Iliadis, \& T. Rauscher, Impact of uncertainties in reaction $Q$ values on nucleosynthesis in type I X-ray bursts, PRC 79 (2009), 045802.

[9] H. Schatz, et al., rp-process nucleosynthesis at extreme temperature and density conditions, Phys. Rep. 294 (1998), 167. 
[10] H. Schatz, et al., The endpoint of the rp-process on accreting neutron stars, Nucl. Phys. A688 (2001), 150.

[11] S. E. Woosley, et al., Models for type I X-ray bursts with improved nuclear physics, ApJS 151 (2004), 75. 